\title{
Adaptação transcultural do Questionário de Comportamentos Agressivos e Reativos entre Pares no Brasil
}

\author{
Juliane Callegaro Borsa - Universidade Federal do Rio Grande do Sul, Porto Alegre, Brasil \\ Denise Ruschel Bandeira - Universidade Federal do Rio Grande do Sul, Porto Alegre, Brasil
}

\begin{abstract}
Resumo
O presente artigo objetiva descrever os procedimentos de tradução e de adaptação transcultural do Questionário de Comportamentos Agressivos e Reativos entre Pares (Q-CARP) para o português brasileiro. Foram realizadas seis etapas para assegurar a qualidade do procedimento: tradução do instrumento do idioma de origem para o idioma-alvo; síntese das versões traduzidas; avaliação por juízes experts; avaliação pelo público-alvo; tradução reversa e estudo piloto. Buscou-se assegurar a qualidade da tradução e da adaptação do Q-CARP no que se refere à equivalência semântica, idiomática, experiencial e conceitual entre os itens originais e traduzidos. A análise da consistência interna das escalas, obtida na etapa do estudo piloto, apresentou correlações satisfatórias entre os itens, demonstrando que estes fazem parte de uma mesma dimensão conceitual. Os resultados indicaram evidências iniciais quanto à adequação do Q-CARP para a avaliação dos comportamentos agressivos e reações ante a agressão em crianças.
\end{abstract}

Palavras-chave: Tradução; Adaptação; Escala; Agressividade.

\section{Cross-cultural adaptation of Peer Aggressive and Reactive Behaviors Questionnaire in Brazil}

\begin{abstract}
The present paper describes the translation and cross-cultural adaptation process of the Peer Aggressive and Reactive Behaviors Questionnaire (PARB-Q) to Brazilian Portuguese. Six steps were performed to ensure the quality of the procedure: two translations from the original language to the target language; synthesis of the translated versions; synthesis evaluation by expert judges; instrument evaluation by the target population; back translation; and pilot study. We sought to guarantee the quality of the translation and adaptation process of the Q-CARP by considering the semantic, idiomatic, experiential and conceptual equivalence of the items between the original and the translated versions. The internal consistencies of the scales were adequate, demonstrating that they are part of the same conceptual dimension. The results indicated initial evidence on the adequacy of the CARP-Q for evaluating aggressive behavior and reactions to aggression in children.

Keywords: Translating; Adaptation; Scale; Aggressiveness.
\end{abstract}

\section{Adaptación transcultural del Cuestionario de Comportamientos Agresivos y Reactivos entre Pares en Brasil}

\section{Resumen}

Este artículo describe los procedimientos de traducción y adaptación transcultural del Cuestionario de Conductas Agresivas y Reactivas entre Pares (Q-CARP) para el portugués brasileño. Fueron realizadas seis etapas para garantizar la calidad del procedimiento: traducción del instrumento del idioma de origen para el idioma de destino; síntesis de las versiones traducidas; evaluación de la síntesis por jueces expertos; evaluación del instrumento por el público objetivo; traducción reversa; y un estudio piloto. Se buscó garantir la calidad de la traducción y de la adaptación del Q-CARP, considerando la equivalencia semántica, idiomática, experiencial y conceptual entre los ítems originales y los traducidos. El análisis de la consistencia interna de las escalas presentó correlaciones satisfactorias entre los ítems, demostrando que estos hacen parte de una misma dimensión conceptual. Los resultados indicaron evidencias iniciales sobre la adecuación del Q-CARP para la evaluación de los comportamientos agresivos y las reacciones frente a las agresiones de los niños.

Palabras-clave: Traducción; Adaptación; Test Psicológico; Agresividad.

\section{Introdução}

O comportamento agressivo é definido como a conduta intencional que tem por objetivo prejudicar ou causar dano físico ou psicológico a uma pessoa ou a um grupo de pessoas (Berkowitz, 1993; Coie \& Dodge, 1998). Trata-se de um problema frequente nas interações entre pares, sobretudo no contexto escolar (Hubbard, McAuliffe, Morrow \& Romano, 2010).

Os comportamentos agressivos têm sido amplamente investigados por pesquisadores das diferentes

Disponivel em wnw.scielo.br áreas em razão de seu impacto negativo tanto para quem agride como para quem sofre a agressão. $\mathrm{Na}$ infância, quando ocorridos de forma intensa e persistente, esses comportamentos configuram-se como fortes preditores de problemas sociais, emocionais e afetivos (Reijntjes e cols., 2011; Temcheff, Serbin \& Martin-Storey, 2011).

$\mathrm{Na}$ interação entre pares, os comportamentos agressivos podem se manifestar de diferentes formas, como agressões físicas (bater, chutar, empurrar, etc.), verbais (gritar, ofender, debochar, etc.) e relacionais 
(difamar, promover intrigas, excluir do grupo social, etc.) (Bjorkqvist, 1994; Crick, 1995; Dodge \& Coie, 1987). Os comportamentos agressivos podem ser classificados, também, como proativos ou reativos (Poulin $\&$ Boivin, 2000). O comportamento agressivo proativo é caracterizado pela agressão deliberada em prol de um objetivo. Crianças que apresentam comportamentos agressivos proativos tendem a priorizar os interesses pessoais em detrimento dos interesses sociais. $\mathrm{O}$ comportamento agressivo reativo, por sua vez, é caracterizado por respostas impulsivas defensivas associadas a sentimentos de raiva e frustração e geralmente ocorre em resposta a uma provocação. Crianças com comportamentos reativos tendem a perceber maior hostilidade nas ações dos seus pares, percebendo-as como provocativas ou agressivas (Crick \& Dodge, 1996; Dodge \& Coie, 1987; Poulin \& Boivin, 2000).

A avaliação dos comportamentos agressivos em crianças é uma prática importante, pois possibilita uma melhor identificação dos sintomas com vistas ao melhor encaminhamento. Para tanto, é fundamental que existam instrumentos que permitam a identificação e o diagnóstico adequado desses comportamentos (Borsa, 2012). Os instrumentos padronizados (escalas, inventários, questionários, entre outros) são comumente utilizados nos procedimentos de avaliação de problemas de comportamento, pois são econômicos, rápidos e fáceis de aplicar. Ademais, os tais instrumentos podem ser importantes ferramentas de screening para uso tanto no contexto clínico quanto escolar.

A literatura internacional conta com diferentes instrumentos para avaliação dos comportamentos agressivos na infância, sendo a escala Teacher-Report Scale (Dodge \& Coie, 1987), uma das mais conhecidas. Trata-se de escala breve composta por seis itens (três para avaliar comportamentos agressivos proativos e três para avaliar os comportamentos agressivos reativos), que inspirou a construção de uma série de outras medidas, como a Revised Teacher Rating Scale of Reactive Aggression and Proactive Aggression (Brown e cols., 1996), a Children's Agression Scale (CAS - Halperin, McKay \& Newcorn, 2002), a Children's Scale of Hostility and Aggression: Reactive/Proactive (C-SHARP - Farmer \& Aman, 2010) e a Parent-Rating Scale Reactive and Proactive Aggression (PRPA - Kempes e cols., 2006).

No Brasil, embora o crescente interesse pelo tema, ainda há poucos instrumentos para a avaliação dos comportamentos agressivos na infância. Borsa e Bandeira (2011) realizaram uma busca por instrumentos adaptados para o contexto brasileiro, destinados a avaliar os comportamentos agressivos de crianças e adolescentes. Para tanto, foram considerados os artigos publicados entre os anos de 1990 e 2010, disponíveis online na Biblioteca Virtual de Psicologia - BVS-Psi (www.bvs-psi.org.br). Foram encontrados onze instrumentos, a maior parte deles para avaliação dos problemas de comportamentos em geral. Dentre os instrumentos específicos para avaliação dos comportamentos agressivos de crianças está a Escala de Agressividade para Crianças e Jovens (Sisto \& Bazi, 2000) e a Escala de Percepção por Professores dos Comportamentos Agressivos de Crianças na Escola (Lisboa \& Koller, 2001). O primeiro é um instrumento de autorrelato, constituído por 16 afirmativas para as quais as crianças devem responder sim ou não (escala dicotômica) quanto à ocorrência de comportamentos agressivos no ambiente familiar e escolar. O segundo instrumento, respondido pela professora, é composto por 41 itens que avaliam os comportamentos agressivos e pró-sociais das crianças na relação com seus pares. Este instrumento ainda não conta com estudos de evidência de validade para o contexto brasileiro.

Os instrumentos encontrados apresentam diferentes propostas e fundamentações teóricas, variando quanto ao tipo de comportamento agressivo avaliado (e.g. físico, verbal, direto e indireto) e quanto ao tipo de informante a que se destina (e.g. autorrelato, pais, professores ou pares). Contudo, nenhum deles avalia, ao mesmo tempo, os comportamentos agressivos proativos e reativos no contexto escolar (Borsa \& Bandeira, 2011). Avaliar os comportamentos agressivos por meio de instrumentos de autorrelato pode se configurar como um recurso importante, sobretudo porque muitos desses comportamentos se manifestam longe da presença de adultos, como os pais e professores (Hyman e cols., 2006).

Uma alternativa para suprir a carência de instrumentos para avaliação de diferentes variáveis psicológicas é adaptar aqueles já existentes em outros contextos. A adaptação de instrumentos psicológicos é uma tarefa complexa que exige planejamento e rigor metodológico (Geisinger, 1994; Hambleton, 1993; Sireci, Yang, Harter, \& Ehrlich, 2006). De acordo com a International Test Comission (ITC, 2010), ao realizar a adaptação de um instrumento para uma nova cultura, é necessário comprovar sua equivalência semântica, idiomática, experiencial e conceitual, bem como suas propriedades psicométricas. 
O termo 'adaptação' tem sido preferido em detrimento do termo 'tradução', pois adaptação compreende todos os procedimentos concernentes à adequação da nova versão do instrumento, para além da mera tradução idiomática (Hambleton, 2005). A tradução é o primeiro passo do processo e precede as outras etapas referentes à adaptação transcultural (Hambleton, 1994, 2005).

Recentemente, Borsa, Damásio e Bandeira (2012) apresentaram uma proposta para o procedimento de tradução e adaptação de instrumentos psicológicos para o contexto brasileiro, a qual é composta por seis etapas: 1) tradução do instrumento do idioma de origem para o idioma-alvo; 2) síntese das versões traduzidas; 3) avaliação da síntese por juízes experts; 4) avaliação do instrumento pelo público-alvo; 5) tradução reversa (back translation) e 6) estudo piloto.

O presente artigo tem como objetivo descrever os procedimentos de tradução e de adaptação do Questionário de Comportamentos Agressivos e Reativos entre Pares (Q-CARP) (Gremigni, Damásio, \& Borsa, 2013) para o português brasileiro, ilustrando, assim, as seis etapas metodológicas propostas por Borsa e cols. (2012). Pretende-se discutir, em específico, as etapas de tradução e de adaptação transcultural dos itens do instrumento - processo que precederá os estudos de evidência de validade a serem realizados consequentemente.

\section{Método}

\section{Participantes}

Para a etapa de tradução do Q-CARP, o estudo contou com a participação de dois juízes bilíngues português-italiano e dois juízes bilíngues português-espanhol. A análise do conteúdo dos itens do Q-CARP contou, ainda, com a participação de profissionais com experiência na área da avaliação psicológica e com conhecimento sobre o construto a ser avaliado pelo instrumento. Também participaram duas crianças (um menino e uma menina, ambos de oito anos, moradores de Porto Alegre e de uma cidade do interior do Rio Grande do Sul, respectivamente), as quais realizaram uma análise qualitativa sobre diferentes aspectos do instrumento. Por fim, para a etapa do estudo piloto, participaram 81 crianças, entre nove e dez anos, sendo 47 meninos $(58 \%)$ e 34 meninas (42\%), residentes na cidade de Porto Alegre, estudantes do quinto ano do ensino fundamental.

\section{Instrumento}

O Q-CARP é um questionário de autorrelato, empiricamente baseado, composto por 20 itens, e que tem por objetivo avaliar os comportamentos agressivos e as reações ante a agressão em crianças no contexto escolar. É composto por duas escalas independentes: a primeira, intitulada Escala de Comportamentos Agressivos (ECA), possui cinco itens e avalia os comportamentos agressivos físicos e verbais, e inclui, também, três itens de controle, os quais não são considerados para pontuação. A segunda escala, intitulada Escala de Reação à Agressão (ERA), é composta por 12 itens que investigam diferentes formas de reação da criança diante dos comportamentos agressivos de seus pares. Possui seis itens referentes às reações agressivas, três itens referentes à busca por apoio do professor e três itens referentes às reações internalizadas caracterizadas por chorar e "ficar emburrado" (Tabela 1).

De origem italiana, o Q-CARP foi construído por Gremigni (2006), a partir de elementos identificados na literatura, incluindo outras medidas de comportamento agressivo infantil, e por meio de grupos de discussões com psicólogos clínicos e escolares e professores do ensino fundamental de escolas públicas e privadas italianas. Na elaboração dos itens, foram consideradas, também, as reações empregadas pela criança (quando em contexto de vitimização) frequentemente observadas no contexto escolar (Gremigni e cols., 2013). A escolha pela validação do Q-CARP, em detrimento de outros instrumentos disponíveis na literatura, ocorreu pela vantagem de avaliar, em um único e breve instrumento, os comportamentos agressivos e as reações empreendidas pela criança frente à agressão dos seus pares.

Análises fatoriais exploratórias (AFE) e confirmatórias (AFC) foram realizadas para avaliar a estrutura do Q-CARP na amostra italiana. A AFE e a análise paralela indicaram solução de um fator para ECA, incluindo os cinco itens com cargas fatoriais maiores que 0,60 e variância explicada de 56,77\%. Já para ERA, a AFE e as análises paralelas indicaram solução de três fatores, quais sejam, Reação Agressiva (RA - 6 itens), Busca de Apoio (BA - 6 itens) e Reação Internalizada (RI - 6 itens), explicando $55,88 \%$ da variância total dos itens. No que se refere à consistência interna dos itens da versão original, a ECA apresentou valor de alfa igual a 0,82. A ERA apresentou três fatores, com índices de consistência interna iguais a 0,86, 0,84 e 0,82, para os fatores RA, BA e RI, respectivamente (para mais detalhes, ver Gremigni e cols., 2013). 
Tabela 1. Itens do Q-CARP nas versões em italiano, espanhol e português

\begin{tabular}{|c|c|c|c|}
\hline & Italiano & Espanhol & Português \\
\hline & Quante volte ti capita di... & Cuántas veces te ocurre... & Quantas vezes acontece de você... \\
\hline 1 & $\begin{array}{l}\text { Dare calci o alzare le mani sui tuoi } \\
\text { compagni? }\end{array}$ & $\begin{array}{l}\text { Dar patadas o levantar la mano a } \\
\text { tus compañeros? }\end{array}$ & $\begin{array}{l}\text { Chutar ou dar um tapa em seus } \\
\text { colegas? }\end{array}$ \\
\hline 2 & Raccontare barzellette? & Contar chistes? & Contar piadas? \\
\hline 3 & $\begin{array}{l}\text { Dire delle brutte cose ai tuoi } \\
\text { compagni? }\end{array}$ & $\begin{array}{l}\text { Decir cosas malas a tus } \\
\text { compañeros? }\end{array}$ & $\begin{array}{l}\text { Dizer coisas ruins para seus } \\
\text { colegas? }\end{array}$ \\
\hline 4 & Essere allegro/a? & Estar alegre? & Ficar alegre? \\
\hline 5 & Prendere in giro i tuoi compagni? & Tomar el pelo a tus compañeros? & Debochar (rir) de seus colegas? \\
\hline 6 & Urlare contro i tuoi compagni? & Gritar a tus compañeros? & Gritar com seus colegas? \\
\hline 7 & Guardare i cartoni in televisione? & Ver los dibujos animados en la tele? & $\begin{array}{l}\text { Assistir desenhos animados na } \\
\text { televisão? }\end{array}$ \\
\hline 8 & $\begin{array}{l}\text { Dare spintoni o graffiare i tuoi } \\
\text { compagni? }\end{array}$ & $\begin{array}{l}\text { Dar empujones o arañar a tus } \\
\text { compañeros? }\end{array}$ & $\begin{array}{l}\text { Empurrar ou arranhar seus } \\
\text { colegas? }\end{array}$ \\
\hline
\end{tabular}

\section{Quando un tuo compagno / a...}

9 Ti dice brutte cose o ti prende in giro, tu gli urli o lo/la tratti male?

10 Ti dà una botta o una spinta, tu lo/ la picchi?

11 Porta via o rompe le tue cose, tu lo/la picchi o rompi le sue cose?

12 Ti dice brutte cose o ti prende in giro, tu ti metti a piangere o metti il muso?

13 Ti dà una botta o una spinta, tu lo dici alla maestra?

14 Porta via o rompe le tue cose, tu gli urli o lo/la tratti male?

\section{Cuando un / a compañero/ a tuyo / a...}

Te dice cosas malas o te toma el pelo, tú le gritas o le tratas mal?

\section{Quando um colega seu...}

Diz coisas ruins, debocha ou ri de você, você grita ou trata mal seu colega?

Bate ou empurra você, você bate no seu colega? pegas?

Se lleva o rompe tus cosas, tú le pegas o rompes sus cosas?

Te dice cosas malas o te toma el pelo, tú te pones a llorar o te pones de morros?

Pega ou estraga alguma coisa sua, você bate no seu colega ou estraga suas coisas?

Diz coisas ruins, debocha ou ri de você, você chora ou fica emburrado (chateado)?

Te da un golpe o un empujón, tú se lo dices a el/la profesor/a?

Bate ou empurra você, você conta para a sua professora?

Pega ou estraga uma coisa sua, você grita ou trata mal o seu colega?

Pega ou estraga uma coisa sua, você conta para a sua professora?

Bate ou empurra você, você chora ou fica emburrado (chateado)? tú te pones a llorar o te pones de morros?

Te dice cosas malas o te Toma el pelo, tú se lo dices a el/la profesor/a?

18 Ti dice brutte cose o ti prende in giro, tu lo/la picchi?

Te dice cosas malas o te toma el pelo, tú le pegas?

19 Porta via o rompe le tue cose, tu ti metti a piangere o metti il muso?

20 Ti dà una botta o una spinta, tu gli urli o lo/la tratti male?
Se lleva o rompe tus cosas, tú te pones a llorar o te pones de morros?

Te da un golpe o un empujón, tú le tratas mal?
Diz coisas ruins, debocha ou ri de você, você conta para a professora?

Diz coisas ruins, debocha ou ri de você, você bate no seu colega?

Pega ou estraga suas coisas, você chora ou fica emburrado (chateado)?

Bate ou empurra você, você grita trata mal o seu colega? 
As análises fatoriais confirmatórias indicaram bons índices de ajuste para a estrutura apresentada em ambas as escalas. As cargas fatoriais padronizadas (pesos de regressão) variaram entre 0,66 e 0,76 para a escala ECA e entre 0,58 e 0,88 para os fatores da ERA. A Figura 1 apresenta a estrutura do instrumento original, suas escalas e seus respectivos fatores.

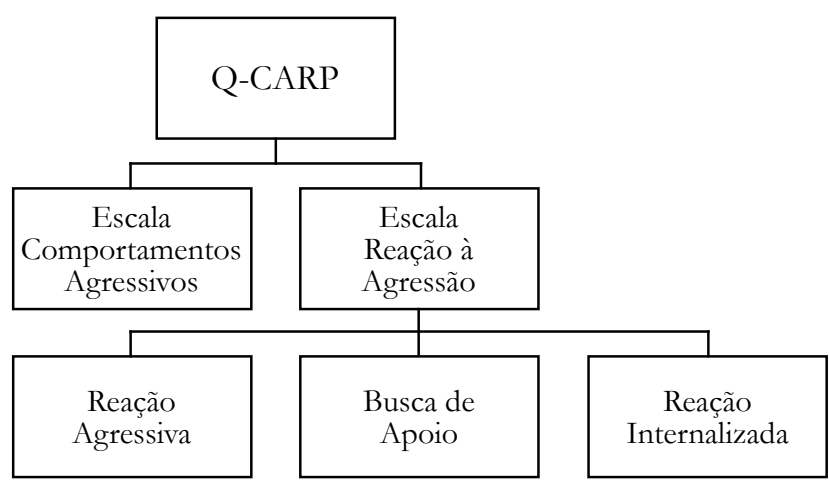

Figura 1. Apresentação do Questionário de Comportamentos Agressivos e Reativos entre Pares - Q-CARP, suas escalas e seus respectivos fatores.

\section{Procedimentos}

Inicialmente, foi feito o contato com a autora do instrumento, por e-mail, e foi solicitada a autorização para a realização da sua tradução e adaptação para o contexto brasileiro. A autorização foi concedida pela autora, que enviou as versões italiana e espanhola do Q-CARP, juntamente com um relatório contendo as informações sobre a construção e validação do instrumento no contexto italiano.

As etapas de tradução e adaptação do Q-CARP para o português brasileiro foram realizadas de acordo com as sugestões de Borsa e cols. (2012), a saber: 1) tradução do instrumento para o novo idioma, 2) síntese das versões traduzidas, 3) avaliação por experts, 4) avaliação pelo público-alvo, 5) tradução reversa (back-translation) e 6) realização do estudo piloto.

A primeira etapa (1) refere-se à tradução do instrumento do idioma de origem para o idioma-alvo. Nela sugere-se que, pelo menos, dois tradutores independentes sejam convidados para traduzir os itens ao novo idioma, verificando a qualidade das traduções e minimizando o risco de possíveis vieses linguísticos, culturais e de compreensão (Beaton e cols., 2000; Hambleton, 2005; ITC, 2010). O processo de síntese das versões traduzidas (2) tem como objetivo comparar as diferentes traduções e avaliar as suas discrepâncias, com o objetivo de se chegar a uma versão única (Borsa e cols., 2012).

Após a elaboração da síntese, o pesquisador convida profissionais com experiência na área da avaliação psicológica e com conhecimento sobre o construto a ser avaliado pelo instrumento para realizar uma avaliação qualitativa (3). Estes irão considerar, por exemplo, o layout, a clareza do rapport, os aspectos da diagramação, a abrangência e adequação das expressões contidas nos itens, entre outros aspectos.

A avaliação pelo público-alvo (4) consiste em apresentar o instrumento a um grupo de juízes-avaliadores com características semelhantes ao público para qual o instrumento se destina (idade, escolaridade, sexo entre outras). Tem como objetivo verificar se os itens, as instruções (rapport) e a escala de respostas são compreensíveis para o público-alvo. Borsa e cols. (2012) sugerem que, em casos de não compreensão de algum item ou termo, os participantes possam sugerir sinônimos que melhor representem o vocabulário usual do grupo de referência.

Após a condução das diferentes etapas concernentes à avaliação do conteúdo do instrumento, realiza-se o procedimento de tradução reversa, também conhecida por back-translation (5). A tradução reversa consiste em traduzir a versão sintetizada e revisada do instrumento para o idioma de origem e tem como objetivo avaliar em que medida a versão traduzida está refletindo o conteúdo da versão original. A tradução reversa é também sugerida como uma verificação de controle de qualidade adicional (Sireci e cols., 2006). Não é objetivo da tradução reversa enfatizar os aspectos gramaticais em detrimento dos aspectos culturais e contextuais (Gudmundsson, 2009; Hambleton, 1993) e sim minimizar possíveis inconsistências ou erros conceituais entre as duas versões (Borsa e cols., 2012).

Por fim, como última etapa do processo de adaptação transcultural, propõe-se a realização do estudo piloto (6). Trata-se de uma aplicação prévia do instrumento em uma pequena amostra que reflita as características da população-alvo (Gudmundsson, 2009). O estudo piloto permite testar a aplicação do instrumento e verificar se existe a necessidade de aprimoramento dos procedimentos metodológicos. A seguir serão descritas as etapas realizadas para a tradução e adaptação do Q-CARP para o contexto brasileiro. 


\section{Resultados e discussão}

\section{Tradução do instrumento}

O primeiro procedimento realizado foi a tradução do Q-CARP do idioma de origem (italiano) para o idioma-alvo (português). Para esta etapa, o estudo contou com a participação de dois tradutores bilíngues português-italiano. Como o instrumento também foi adaptado na Espanha, solicitou-se, como um recurso adicional, que dois tradutores bilíngues português-espanhol realizassem a tradução da versão espanhola para o português.

Aos tradutores foi solicitado que evitassem regionalismos e expressões locais que pudessem ser usuais em um contexto do Brasil e pouco conhecidos em outros. Não foram observadas, ao longo deste processo, discrepâncias entre as traduções oriundas da versão italiana e espanhola do Q-CARP. Assim, todos os itens foram mantidos iguais aos originais. A Tabela 1 apresenta os itens em suas diferentes versões em italiano, espanhol e português.

\section{Sintese das versões traduzidas}

Após a realização das traduções, obteve-se quatros versões em português do Q-CARP (duas oriundas da versão em italiano e duas oriundas da versão em espanhol). A partir dessas, foi realizado o processo de síntese, o qual consistiu em comparar as diferentes traduções e avaliar as suas possíveis discrepâncias, com o objetivo de se chegar a uma única versão (Borsa e cols., 2012). O processo de síntese foi realizado pelas pesquisadoras responsáveis pelo estudo de tradução e adaptação transcultural do instrumento no Brasil.

A avaliação das diferentes traduções foi realizada para cada item em particular. As pesquisadoras avaliaram a equivalência entre as versões traduzidas e o instrumento original, no que se refere aos seguintes aspectos: equivalência semântica (se as expressões apresentam o mesmo significado e se existem erros gramaticais na tradução); equivalência idiomática (se os itens mais difíceis ou dúbios foram adaptados adequadamente); equivalência experiencial (se os itens e termos de um instrumento são aplicáveis na nova cultura); e equivalência conceitual (se determinado termo, mesmo que traduzido adequadamente, avalia o mesmo aspecto no novo contexto quando comparado ao contexto da versão original). Assim, ao final desta etapa, as pesquisadoras passaram a contar com uma única versão do Q-CARP (Borsa e cols., 2012).
Avaliação por experts

Com o intuito de avaliar conteúdo e forma (layout, rapport, diagramação, clareza e adquação dos itens, entre outros), solicitou-se a avaliação da versão sintetizada do Q-CARP por um grupo de psicólogos (todos mestrandos e doutorandos) com experiência na adaptação transcultural de instrumentos psicológicos e na avaliação psicológica clínica de crianças. Para a realização desta etapa, foi conduzida uma atividade em grupo para apresentar a estrutura do instrumento, seus objetivos e público-alvo. Após esta explanação, foi entregue a cada participante uma ficha para que respondessem aos seguintes critérios: $\mathrm{O}$ item é pertinente para avaliar o construto? O item está bem formulado? O item está claro? O item deve ser modificado? Se sim, qual sua sugestão de modificação para o item?

\section{Avaliação pelo público-alvo}

Esta etapa do processo teve por objetivo verificar se os itens, as instruções e a escala de respostas são compreensíveis para o público-alvo. Para tanto, duas crianças (um menino e uma menina, ambos de oito anos), foram convidados a realizar a análise qualitativa sobre diferentes características do instrumento, observando detalhes como clareza dos termos (tanto dos itens quanto do rapport) e adequação das expressões.

Borsa e cols. (2012) recomendam que, em casos de não compreensão de algum item ou termo, os participantes podem sugerir sinônimos que melhor representem o vocabulário usual do grupo de referência. No caso do Q-CARP, solicitou-se às crianças que sublinhassem os termos julgados difíceis ou incompreensíveis, e para estes realizou-se uma explicação sobre seu significado. Após, perguntou-se "Agora que você já entendeu o que esta palavra significa, que outra palavra você usaria para dizer a 'mesma coisa'?'. Assim, as crianças sugeriram sinônimos ou palavras substitutas, mas de mesmo sentido. Os termos sugeridos foram inseridos em parênteses, ao lado dos termos originais do questionário. Os termos originais que apresentaram dificuldade de compreensão, por parte das crianças, e os sinônimos sugeridos foram: 1) Debochar (rir); 2) Magoar (chatear); 3) Emburrado (chateado).

\section{Tradução reversa}

Finalmente, após os procedimentos acima citados, a versão preliminar do Q-CARP foi traduzida para o idioma italiano. Esses procedimento foi realizado por um terceiro tradutor bilíngue e nativo no idioma italiano, o qual não participou da primeira etapa de 
tradução. A versão traduzida foi enviada à autora do instrumento original e também da versão espanhola. A ela foi explicado o motivo da inserção dos termos sinônimos em parênteses. Também foi realizada uma explanação sobre as características culturais do Brasil e sobre a importância dos itens serem claros para crianças pertencentes a diferentes contextos. Por fim, a versão final do instrumento, incluindo as modificações realizadas nos itens e no layout, foi autorizada pela autora. O procedimento de tradução reversa do Q-CARP foi avaliado positivamente, uma vez que permitiu a realização de um brainstorm entre os autores, com vistas a assegurar a equivalência entre os itens das duas versões, respeitando as diferenças culturais de cada contexto.

\section{Estudo piloto}

O estudo piloto teve como objetivo testar a aplicação do Q-CARP. Esta etapa contou com a participação de 81 crianças, entre nove e dez anos, sendo 47 meninos $(58 \%)$ e 34 meninas (42\%), residentes na cidade de Porto Alegre, estudantes do quinto ano do ensino fundamental. A coleta de dados aconteceu em uma escola da região central da cidade de Porto Alegre, Rio Grande do Sul. A aplicação dos instrumentos aconteceu coletivamente, em sala de aula, na presença de dois pesquisadores previamente treinados. O tempo médio para preenchimento do instrumento pelas crianças foi de quinze minutos. As questões éticas da pesquisa foram asseguradas, conforme Resolução n 196/96, do Ministério da Saúde (Brasil, 1996). Do mesmo modo, todos os procedimentos atenderam às exigências do Comitê de Ética e Pesquisa do Instituto de Psicologia da UFRGS.

Para a análise preliminar dos dados, foi calculada a consistência entre os itens, para cada uma das escalas do Q-CARP, mediante o alfa de Cronbach. O objetivo foi verificar se, de fato, os itens do instrumento possuíam correlações entre si (uma vez que medem o mesmo construto). Quanto maior a homogeneidade do conteúdo referente aos itens, maior será a consistência interna do instrumento (Cronbach, 1996).

A análise da consistência interna das escalas do Q-CARP evidenciou correlações satisfatórias entre os itens, indicando que estes fazem parte de uma mesma dimensão conceitual: $\mathrm{ECA}=0,73 ; \mathrm{RA}=0,85 ; \mathrm{BA}=$ 0,82 e RI $=0,82$. Os valores de alfa de Cronbach obtidos para esta amostra foram semelhantes ao encontrado no estudo original (Gremigni e cols., 2013). Por fim, após última etapa, deu-se por finalizado o processo de tradução e de adaptação transcultural dos itens do Q-CARP.

\section{Considerações finais}

O objetivo deste estudo foi descrever os procedimentos metodológicos de tradução e de adaptação do Questionário de Comportamentos Agressivos e Reativos entre Pares - Q-CARP (Gremigni e cols., 2013) para o português brasileiro. Para este fim, seguiram-se algumas etapas fundamentais para a adequada realização do processo, conforme sugerido por diferentes pesquisadores (Beaton e cols., 2000; Gjersing e cols., 2010; Hambleton, 2005) e sintetizados por Borsa e cols. (2012).

Entende-se que a qualidade da tradução e da adaptação transcultural de um instrumento psicológico apresenta um papel fundamental para garantir que os resultados obtidos em pesquisas transculturais não se devam a erros de tradução, mas sim, às reais diferenças ou similaridades entre os grupos em que determinado fenômeno está sendo mensurado. Neste sentido, este estudo buscou primar pelo adequado procedimento de tradução e adaptação do Q-CARP, considerando a pertinência dos conceitos e domínios apreendidos pelo instrumento original, bem como considerando as equivalências semântica, idiomática, experiencial e conceitual entre os itens originais e os itens traduzidos.

Assumindo, a priori, a estrutura fatorial do instrumento original italiano e de sua versão espanhola, verificou-se que o Q-CARP apresentou bons coeficientes alfa tanto para a ECA (unidimensional), como para os três fatores (RA, BA e RI) da ERA. Os itens apresentaram-se compreensíveis para as crianças participantes do estudo piloto, indicando que as alterações e a inclusão de sinônimos entre parênteses mostraram-se adequadas.

É importante salientar que a tradução e a adaptação são apenas as primeiras etapas do processo de validação de um instrumento psicológico para um novo contexto cultural. Assim, novos estudos são necessários a fim de buscar outras evidências de validade do Q-CARP no Brasil. Análises fatoriais exploratórias (AFEs) e confirmatórias (AFCs) permitirão avaliar a plausibilidade da estrutura fatorial das escalas do Q-CARP. As técnicas de funcionamento diferencial do item (em inglês, differential item functioning, DIF), da Teoria de Resposta ao Item (TRI), poderão ser úteis para avaliar a similaridade dos itens do instrumento para diferentes grupos (Sireci e cols., 2006). Evidências de validade baseadas na relação com outras variáveis (validade convergente, externa, discriminante, entre outras), poderão ser obtidas mediante a comparação 
dos escores do Q-CARP com os resultados de outros instrumentos reconhecidos como 'padrão-ouro' e que avaliam o mesmo construto ou construtos correlatos (AERA, APA \& NCME, 1999).

\section{Referências}

AERA - American Educational Research Association, APA - American Psychological Association, \& NCME - National Council on Measurement in Education. (1999). Standards for Educational and Psychological Testing. Nova Iorque: American Educational Research Association.

Beaton, D. E., Bombardier, C., Guillemin, F., \& Ferraz, M. B. (2000). Guidelines for the process of cross-cultural adaptation of self-report measures. Spine, 25(24), 3186-3191.

Berkowitz, L. (1993). Aggression: its causes, consequences, and control. Nova Iorque: McGraw-Hill.

Björkqvist, K. (1994). Sex differences in physical, ver$\mathrm{bal}$, and indirect aggression: a review of recent research. Sex Roles, 30(3-4), 177-188.

Borsa, J. C. (2012). Adaptação e validação transcultural do Questionário de Comportamentos Agressivos e Reativos entre Pares (Q-CARP) (Tese de Doutorado não publicada). Universidade Federal do Rio Grande do Sul, Porto Alegre, Brasil.

Borsa, J. C., \& Bandeira, D. R. (2011). Uso de instrumentos psicológicos de avaliação do comportamento agressivo infantil: análise da produção científica brasileira. Avaliação Psicológica, 10(2), 193-203.

Borsa, J. C., Damásio, B. F., \& Bandeira, D. R. (2012). Adaptação e validação de instrumentos psicológicos entre culturas: algumas considerações. Paidéia, 22(53), 423-432.

Brasil (1996). Ministério da Saúde. Conselho Nacional de Saúde. Comissão Nacional de Ética em Pesquisa-CONEP. Resolução no. 196/96. Dispõe sobre pesquisa envolvendo seres humanos. Ministério da Saúde, Brasilia.

Brown, K., Atkins, M. S., Osborne, M. L., \& Milnamow, M. (1996). A revised teacher rating scale for reactive and proactive aggression. Journal of Abnormal Child Psychology, 24(4), 473-480.

Coie, J. D., \& Dodge, K. A. (1998). Aggression and antisocial behavior. Em W. Damon \& N. Eisenberg
(Eds.), Handbook of child psychology: social, emotional, and personality development ( $5^{\text {th }}$ ed., pp. 779-862). Toronto, Canada: John Wiley \& Sons.

Crick, N. R. (1995). Relational aggression: the role of intent attributions, feelings of distress, and provocation type. Development and Psychopathology, 7(2), 313-322.

Crick, N. R., \& Dodge, K. A. (1996). Social informationprocessing mechanisms in reactive and proactive aggression. Child Development, 67(3), 993-1002.

Cronbach, L. J. (1996). Fundamentos da testagem psicológica (5 $5^{\mathrm{a}}$ ed.). Porto Alegre: Artmed.

Dodge, K. A., \& Coie, J. D. (1987). Social-information-processing factors in reactive and proactive aggression in children's peer groups. Journal of Personality and Social Psychology, 53(6), 1146-1158.

Farmer, C., \& Aman, M. (2009). Development of the Children's Scale of Hostility and Aggression: reactive/proactive (C-SHARP). Research in Developmental Disabilities, 30(6), 1155-1167.

Geisinger, K. F. (1994). Cross-Cultural normative assessment: translation and adaptations issues influencing the normative interpretation of assessment instrument. Psychological Assessment, 6(4), 304-312.

Gjersing, L., Caplehorn, J. R. M., \& Clausen, T. (2010). Cross-cultural adaptation of research instruments: language, setting, time and statistical considerations. BMC Medical Research Methodology, 10(1), $10-13$.

Gremigni, P. (2006). Validazione di un nuovo Questionario Per L'eta' Evolutiva Il Cap Comportamento Aggressivo Tra Pari. Report di Ricerca. Universita' Degli Studi di Bologna, Dipartimento di Psicologia (manuscrito não-publicado).

Gremigni, P., Damásio, B. F., \& Borsa, J. C. (2013). Development and validation of a questionnaire to evaluate overt aggression and reactions to peer aggression. Psicologia: Reflexão e Crítica, 26(2), 311-318.

Gudmundsson, E. (2009). Guidelines for translating and adapting psychological instruments. Nordic Psychology, 61(2), 29-45.

Halperin, J. M., McKay, K. E., \& Newcorn, J. H. (2002). Development, reliability and validity of the Children's Aggression Scale-Parent Version. Journal of 
the American Academy of Child \& Adolescent Psychiatry, 41(3), 245-252.

Hambleton, R. K. (1993). Translating achievement tests for use in cross-national studies. European Journal of Psychological Assessment, 9(1), 57-68.

Hambleton, R. K. (1994). Guidelines for adapting educational and psychological tests: a progress report. European Journal of Psychological Assessment, 10(3), 229-244.

Hambleton, R. K. (2005). Issues, designs and technical guidelines for adapting tests into multiple languages and cultures. Em R. K. Hambleton, P. F. Merenda, \& C. D. Spielberger (Eds.), Adapting educational and psychological tests for cross-cultural assessment (pp. 3-38). Mahwah, N. J.: Erlbaum.

Hubbard, J. A., McAuliffe, M. D., Morrow, M. T., \& Romano, L. J. (2010). Reactive and proactive aggression in childhood and adolescence: precursors, outcomes, processes, experiences, and measurement. Journal of Personality, 78(1), 95-118.

Hyman, I., Kay, B., Tabori, A., Weber, M., Mahon, M., \& Cohen, I. (2006). Bullying: theory, research and intervention. Em C. M. E. Evertson \& C. S. E. Weinstein (Eds.), Handbook of classroom management: research, practice, and contemporary issues (pp. 855-884). Mahwah, NJ: Lawrence Erlbaum.

ITC - International Test Commission (2010). ITC Guidelines of Adapting Tests. Disponível: http:// www.intestcom.org.

Kempes, M., Matthys, W., Maassen, G., \& van Engeland, H. (2006). A parent questionnaire for distinguishing between reactive and proactive aggression in children. European Child and Adolescent Psychiatry 15(1), 38-45.

Lisboa, C. S. M., \& Koller, S. H. (2001). Construção e validação de conteúdo da Escala de Percepção de Professores dos Comportamentos Agressivos de Crianças na Escola. Psicologia em Estudo, 6(1), 59-69.

Poulin, F., \& Boivin, M. (2000) Reactive and proactive aggression: evidence of a two-factor model. Psychological Assessment, 12(2), 115-122.

Reijntjes, A., Kamphuis, J. H., Prinzie, P., Boelen, P. A, van der Schoot, M., \& Telch, M. J. (2011). Prospective linkages between peer victimization and externalizing problems in children: a meta-analysis. Aggressive Behavior, 37(3), 215-222.

Sireci, S. G., Yang, Y., Harter, J., \& Ehrlich, E. J. (2006). Evaluating guidelines for test adaptations: a methodological analysis of translation quality. Journal of Cross-Cultural Psychology, 37(5), 557-567.

Sisto, F. F., \& Bazi, G. A. P. (2000). Escala de Agressividade para crianças e jovens (relatório técnico). Faculdade de Educação: UNICAMP.

Temcheff, C. E., Serbin, L. A., \& Martin-Storey, A. (2011). Predicting adult physical health outcomes from childhood aggression, social withdrawal and likeability: a 30-year prospective, longitudinal study. International Journal of Behaviorl Medicine, 18(1), 5-12.

Recebido em: 05/04/2013

Reformulado em: 22/10/2013

Segunda reformulação em: 10/12/2013

Aprovado em: 03/02/2014 
Sobre as autoras:

Juliane Callegaro Borsa é professora do Departamento de Psicologia e coordenadora adjunta do Programa de Pós-Graduação em Psicologia Clínica da Pontifícia Universidade Católica do Rio de Janeiro (PUC-Rio). Mestre em Psicologia Clínica pela Pontifícia Universidade Católica do Rio Grande do Sul (PUCRS), doutora e pós-doutora em Psicologia pela Universidade Federal do Rio Grande do Sul (UFRGS), coordena o grupo de pesquisa AP-Lab: Avaliação Psicológica, Pessoas e Contextos.

Denise Ruschel Bandeira é graduada em Psicologia pela Pontifícia Universidade Católica do Rio Grande do Sul (1988), mestre em Psicologia pela Universidade Federal do Rio Grande do Sul (1991) e doutora em Psicologia pela Universidade Federal do Rio Grande do Sul (2000). É a atual coordenadora do Programa de Pós-Graduação em Psicologia UFRGS, orientadora de mestrado e doutorado e coordenadora do Grupo de Estudos, Aplicação e Pesquisa em Avaliação Psicológica.

\section{Contato com as autoras:}

Juliane Callegaro Borsa

Rua Marquês de São Vicente, 225, Edifício Cardeal Leme, sala 201

Gávea, Rio de Janeiro, RJ. CEP 22453-900

E-mail: juliborsa@gmail.com 